\title{
Integrated Real-Time Digital Measurement During Ureteroscopic Procedures for Nephrolithiasis: A Workflow Feasibility Study
}

\author{
Kevin Koo, MD, MPH, MPhil, ${ }^{1}$ Tareq Aro, MD,1,2 Russell E.N. Becker, MD, PhD, ${ }^{1}$ Sunghwan Lim, PhD, ${ }^{1,2}$ \\ Jared S. Winoker, MD, Doru Petrisor, $\mathrm{PhD}^{1,2}$ Dan Stoianovici, $\mathrm{PhD}^{1,2}$ and Brian R. Matlaga, MD, MPH ${ }^{1}$
}

\begin{abstract}
Introduction: Accurate estimation of stone fragment size during ureteroscopic lithotripsy procedures facilitates operative efficiency and predicts the safety of fragment extraction or spontaneous passage. Using a novel stone measurement software application, this study assesses the feasibility of performing integrated real-time digital stone measurement during ureteroscopy.

Methods: This workflow feasibility study was conducted in two phases. First, in the ex vivo simulation, mock stone fragments were placed in a renal collecting system training model. A basket extraction task was performed using a digital ureteroscope, with and without digital stone measurement. The time required to perform the tasks was recorded and compared. Second, in the in vivo workflow trial, adult patients undergoing ureteroscopic stone procedures were prospectively enrolled. Intraoperative measurements of stone fragments were performed to determine the time required to complete the measurements. Technical failures and perioperative complications were recorded.

Results: In the ex vivo simulation, 20 mock stones mimicking varied fragmentation conditions were tested in the training model. The mean completion time of the basketing task without $v s$ with digital stone measurement was 16.5 seconds (range 10.2-33.7) vs 38.9 seconds (range 27.2-60.0). Mean additional time required to measure stones was 22.3 seconds (range 8.4-42.7). In the in vivo workflow trial, nine patients undergoing ureteroscopy were enrolled. A median of five fragments (range 3-10) were measured in each patient. Mean completion time for each measurement was 10.1 seconds (range 8.2-12.8). Mean total time required to perform digital measurement per procedure was 50.8 seconds (range 25.9-99.0). No technical failures or clinical complications were observed.

Conclusions: Integrating real-time digital stone measurement during ureteroscopy is safe and feasible. The findings support clinical trials of digital stone measurement to enhance intraoperative decision-making during ureteroscopy.
\end{abstract}

Keywords: ureteroscopy, software, nephrolithiasis, measurement, lithotripsy

\section{Introduction}

A CCURATE ESTIMATION OF STONE fragment size is an important aspect of ureteroscopic lithotripsy procedures in the endoscopic management of nephrolithiasis. Incomplete lithotripsy may increase operative time and disrupt workflow by requiring further rounds of fragmentation with repeated exchanges between the laser fiber and extraction basket. Reliable determination of fragment size promotes efficient continuous lithotripsy and uninterrupted extraction. In addi- tion, fragment size also predicts the likelihood of spontaneous passage. Leaving large fragments behind increases the potential risk of ureteral injury during basket extraction or postoperative obstruction. ${ }^{1,2}$ Measurement of stone fragments during ureteroscopic procedures can thus provide real-time guidance to the surgeon regarding completion of lithotripsy and extractability of fragments.

Few hardware tools and software applications are available, however, to aid surgeons in measuring stones during live ureteroscopy. Surgeons may make cognitive estimates

\footnotetext{
${ }^{1}$ Department of Urology, Johns Hopkins University School of Medicine, Baltimore, Maryland, USA
}

${ }^{2}$ Robotics Laboratory, Johns Hopkins University School of Medicine, Baltimore, Maryland, USA. 
based on the known sizes of anatomic landmarks or surgical devices (e.g., laser fiber tip), but these estimates may be unreliable or inconsistent. ${ }^{3,4}$ A novel software application has been developed to determine the linear length of an object using a single-use digital ureteroscope. ${ }^{5}$ This tool is accurate to $0.15 \mathrm{~mm}$, with longitudinal and transverse error margins $<0.2 \mathrm{~mm}$. Initial experiments using the software were performed on recorded videos of ureteroscopy procedures. The feasibility of integrating stone measurement into operating room workflow is not known.

The aim of this study was to investigate the feasibility of performing real-time digital stone measurement during live ureteroscopy. We conducted a workflow feasibility trial to determine the additional operative time needed to integrate the stone measurement software application into clinical settings, as well as the safety considerations of software integration.

\section{Methods}

We conducted the study in two phases: an ex vivo simulation and an in vivo workflow trial.

In the ex vivo simulation, a series of mock stones was placed in the superior pole of a commercial flexible ureteroscopy training model. For each stone, a trained endourologist used a single-use digital flexible ureteroscope (LithoVue ${ }^{\mathrm{TM}}$; Boston Scientific) with the measurement software to perform two basketing tasks, a conventional version and a measurement version. In the conventional version of the basketing task, we navigated the ureteroscope from the ureteropelvic junction (UPJ) to the stone in the superior pole, snared it with a stone extraction basket (Zero Tip ${ }^{\mathrm{TM}}$; Boston Scientific), then retrieved it to the UPJ. In the measurement version of the basketing task, we repeated the same process, but before snaring the stone, we measured the size of the stone using the software in real time, followed by retrieval to the UPJ. Total completion time for each iteration of the task was measured for all stones.

In the in vivo workflow trial, the aim was to determine whether digital measurements of stone fragments could be completed in real time during surgery, as well as the time required to perform the measurements. We prospectively enrolled adult patients, age $>18$ years, undergoing elective ureteroscopy and laser lithotripsy for renal or ureteral stones by two trained endourologists. We used a new single-use digital flexible ureteroscope of the same model to perform ureteroscopy. Lithotripsy was performed per surgeon preference.

At the completion of lithotripsy and before fragment extraction, one or more stone fragments were selected by the surgeon to be measured using the software. This process has been previously described. ${ }^{5}$ The ureteroscope was navigated to capture the fragment in full view. Each fragment was snared by the stone extraction basket of the same model, then a screen capture was performed, allowing digital measurements to be taken of the fragment in the longitudinal dimension. The software was digitally calibrated before the procedure to correct for small variations in distance between the tip of the ureteroscope and the fragment. ${ }^{5}$ The edges of each target fragment were identified immediately before measurement to avoid including adjacent fragments in the calculation. Once the measurement had been completed, the surgeon continued with the procedure. The time required to perform each measurement or series of measurements was recorded, including initiation of the digital measurement by the surgeon, screen capture, and confirmation of size calculation. Software-related failures, perioperative complications, and delays were also recorded. To avoid the potential for the measurements to influence clinical decision-making, surgeons were blinded to the results of the measurements during the procedure. However, all fragments that were measured were selected by the surgeon with the intention of supporting clinical decisions.

Institutional review board approval was obtained for the in vivo workflow trial (protocol IRB00185627).

\section{Results}

In the ex vivo simulation, 20 mock stones with a variety of internal characteristics were selected to mimic varied fragmentation conditions. Mean stone size was $7.1 \mathrm{~mm}$ (range $3.2-10.3 \mathrm{~mm}$ ). Different primary three-dimensional shapes were represented: 10 stones (50\%) were pyramidal, 6 stones (30\%) were ovoid, and 4 stones (20\%) were cuboid. Surface contour of the stones was divided equally between smooth and rough (10 stones in each group, 50\%). In terms of planar symmetry, 8 stones $(40 \%)$ were symmetric, whereas 12 stones $(60 \%)$ were asymmetric.

In the conventional basketing task involving navigation to the stone, snaring the stone with a basket, and retrieval to the UPJ, the mean completion time of a single trial was 16.5 seconds (range 10.2-33.7, SD 5.8). In the measurement basketing task involving navigation to the stone, real-time measurement of the stone using the software, snaring the stone, and retrieval, the mean completion time of a single trial was 38.9 seconds (range 27.2-60.0, SD 8.7). The mean additional time required to perform real-time digital stone measurement was 22.3 seconds (range 8.4-42.7, SD 8.7). There were no failures of the software during any of the trials. Figure 1 contains representative examples of the stones and screen capture during the measurement basketing task.

In the in vivo workflow trial, we performed real-time stone measurement during ureteroscopic stone procedures. A total of nine patients undergoing elective ureteroscopy participated in the trial. Characteristics of the patients and stones are summarized in Table 1. After laser lithotripsy, intraoperative real-time measurement of stone fragments was completed in all patients. For each patient, a median of five measurements (range 3-10) were taken. Measurements were performed for all attempts. The mean completion time for each measurement, from snaring the fragment until size calculation, was 10.1 seconds (range 8.2-12.8, SD 1.7). The mean total time spent performing digital measurement per procedure was 50.8 seconds (range 25.9-99.0, SD 24.2). Figure 2 contains screen captures during the real-time measurements.

There were no technical failures of the software or ureteroscope. No measurement-related delays or perioperative clinical complications were observed. Mean completion time did not vary significantly based on location of the stones or the number of repeated measurements performed $(p>0.05)$.

\section{Discussion}

Ureteroscopic lithotripsy is commonly performed in the contemporary management of nephrolithiasis, both in the 

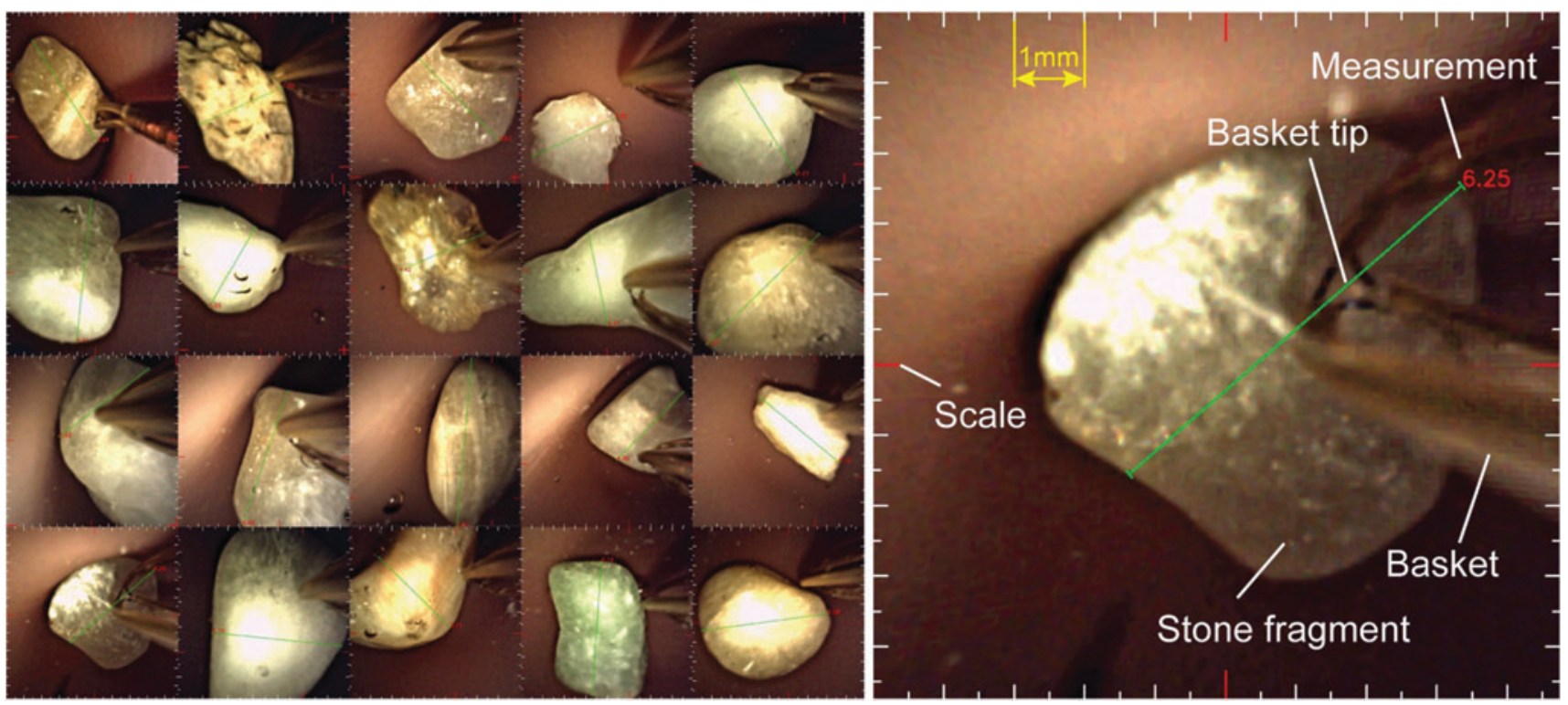

FIG. 1. Ex vivo simulation of digital stone measurement during ureteroscopy. Left: Examples of mock stones with varied size, shape, contour, and planar symmetry. Right: Representative screen capture showing extraction basket tip relative to stone and digital measurement of stone dimension. Measurement (red) in millimeters.

United States ${ }^{6}$ and worldwide, ${ }^{7}$ in part because ureteroscopy is recommended for a broad range of stone sizes and locations in clinical practice guidelines. ${ }^{8,9}$ The ability to determine stone fragment size after lithotripsy can inform surgeons regarding the completion of fragmentation and the safety of extraction through the ureter. In this workflow feasibility study, we demonstrated in both an ex vivo stimulation and an in vivo clinical environment that integrated real-time digital measurement can be performed during ureteroscopy procedures with no added risk to the patient and minimal additional operative time. The software reliably calculated the measurements for a variety of stone shapes, dimensions, and locations in the upper tract, without disruption to the procedural workflow or occurrence of perioperative complications.

This is the first report of real-time integrated ureteroscopic stone measurement using a digital measurement software application. The initial study of the software relied on recorded videos to calculate stone size and determine accuracy and reliability of the software measurements compared with caliper measurements of the fragments. ${ }^{5}$ This study builds on the prior findings by confirming the ability to perform digital measurements in a live operating room setting without significant additional time. Recognizing the importance and utility of endoscopic stone measurement, recent innovations in basket design have attempted to integrate a measurement tool into the basket itself using a handle-based scale. ${ }^{10}$ An initial prototype reported $84 \%$ specificity to predict stones $>6 \mathrm{~mm}$, but it was less accurate than visual estimates by surgeons. ${ }^{11}$ A newer model included standardized millimeter markings on the basket handle to gauge stone size based on the degree of closure of the basket ${ }^{12}$; the accuracy of this method is not yet known. In contrast to these methods, the digital measurement technique in this study directly measures stone fragments based on their endoscopic appearance and has been demonstrated to be more accurate than expert surgeons' visual estimates. ${ }^{4}$ An additional advantage is that the digital ureteroscope platform accommodates the novel software without additional cost or modification of the extraction basket, and our method for stone measurement may be used in any clinical situation in which this digital ureteroscope is appropriate. ${ }^{5}$ Whether digital or mechanical

Table 1. Patient and Case Characteristics for the IN Vivo Workflow Trial

\begin{tabular}{rrlllcrcr}
\hline Patient & $\begin{array}{c}\text { Age } \\
\text { (years) }\end{array}$ & Gender & Laterality & $\begin{array}{c}\text { Measurement } \\
\text { location }\end{array}$ & $\begin{array}{c}\text { Number of } \\
\text { measurements } \\
\text { performed }\end{array}$ & $\begin{array}{c}\text { Total } \\
\text { measurement } \\
\text { time (seconds) }\end{array}$ & $\begin{array}{c}\text { Mean time } \\
\text { per measurement } \\
\text { (seconds) }\end{array}$ \\
\hline 1 & 75 & Female & Right & Kidney, pelvis & 6 & 77.0 & 12.8 \\
2 & 28 & Female & Left & Kidney, interpolar & 5 & 44.0 & 8.8 \\
3 & 63 & Male & Right & Kidney, pelvis & 10 & 99.0 & 11.0 \\
4 & 46 & Male & Right & Kidney, superior pole & 3 & 33.0 & 10.0 \\
5 & 27 & Female & Right & Ureter, proximal & 5 & 50.0 & 12.6 \\
6 & 62 & Female & Left & Ureter, proximal & 4 & 30.2 & 8.6 \\
7 & 64 & Female & Left & Kidney, pelvis & 3 & 25.9 & 8.2 \\
8 & 25 & Female & Right & Kidney, pelvis & 3 & 24.7 & 8.9 \\
9 & 50 & Male & Left & Kidney, inferior pole & 6 & 53.3 & \\
\hline
\end{tabular}



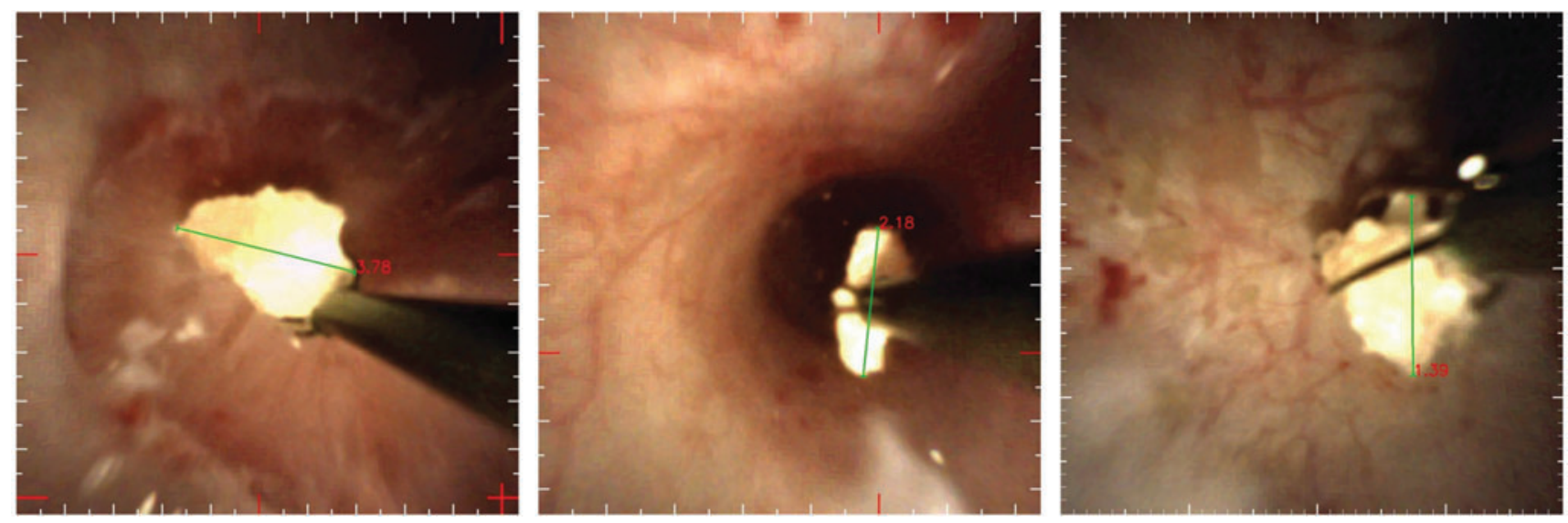

FIG. 2. Digital stone measurement during in vivo ureteroscopy. Representative images of real-time digital stone measurement after lithotripsy at the interpolar region (left), inferior pole (middle), and renal pelvis (right). Measurements (red) in millimeters.

approaches to real-time measurement prove to be more accurate and easily integrated into endoscopic procedures merits further study.

We observed variation in the additional time required to complete the digital measurement during the ex vivo basketing tasks, ranging from 8 to 43 seconds, despite a fixed superior pole location and controlled settings. There are several potential explanations for the observed variation. Different mock stone characteristics in some cases resulted in challenging planar alignment of the ureteroscope and basket tip. Greater technical manipulation was required for larger mock stones, in particular those $>7 \mathrm{~mm}$, because the widest dimension had to be captured in the endoscopic field of view before measurement. In clinical settings, however, it would be unlikely for fragments of this size to warrant endoscopic measurement, as they generally would not be considered suitable for basket extraction or spontaneous passage. Finally, the response of the ureteroscopy training model may have been different compared with live tissues. This was confirmed in the in vivo trial, in which we noted more consistent mean completion times (8.2-12.8 seconds).

This study has several limitations. First, in the context of a small feasibility trial, we recruited a limited sample of patients, so we did not perform measurements systematically at every renal location. We did, however, take measurements in a variety of sites (Table 1) and did not observe significant differences in the time required to measure the fragments among the sites. Second, measurement data were not disclosed to the surgeon during the procedure as required by the local institutional review board to avoid impact on intraoperative decision-making during this trial. Thus, we were not able to determine whether performing real-time stone measurement could result in appreciable time or cost savings. Third, we cannot directly compare the calculated completion times between the two phases of the study. In the ex vivo phase the time included navigation of the ureteroscope to and from a standardized location, whereas during live ureteroscopy we determined the time to perform the measurement itself while maintaining the ureteroscope in place. However, because of the inclusion of ureteroscope navigation, we expect that the completion times from the ex vivo phase would be overestimations, suggesting that the actual additional time of performing digital measurements is relatively modest. Fourth, our software application was optimized for and tested using the endoscopic basket as a standardized scaling calibration instrument. The algorithm could in theory allow for a laser fiber or another endoscopic device to be used for calibration. Future software iterations will expand this flexibility to improve intraoperative integration and user experience. Finally, our software was calibrated for use with a specific model of a digital ureteroscope. We achieved reliable results using a new ureteroscope for each case, but we did not assess other single-use or reusable ureteroscopes, although we would expect the potential advantages of real-time stone measurement to be similar.

Despite these limitations, the study has important implications. First, the findings have confirmed that real-time digital measurement of stone fragments is feasible and can be performed during typical operating room workflow with modest additional time. The next step is to incorporate selective endoscopic measurement to support intraoperative decisions to extract fragments or leave them safely behind, which may address several questions. Does stone measurement facilitate improved efficiency by avoiding occasions when the stone is found to be too large to basket or extract through a ureteral access sheath, necessitating multiple instrument exchanges or laser rescue procedures? ${ }^{13,14}$ In cases when stones are dusted, can stone measurement help to confirm particle size below a $1 \mathrm{~mm}$ threshold and reduce the incidence of Steinstrasse or large residual fragments on postoperative imaging? ${ }^{15}$ Examining lithotripsy efficiency (e.g., fewer exchanges between laser fiber and extraction basket), procedural cost (e.g., fewer disposables), stone clearance or fragmentation completion, operative time, injury and complications, and impact on surgeons' decisionmaking (e.g., subjective usefulness of quantitative stone measurement) in a trial setting will help characterize the contexts in which digital measurement provides actionable information. Technical automation of the screen capture and digital measurement processes may also reduce the time required and enhance clinical application.

Second, real-time endoscopic measurement has numerous potential applications beyond stone fragments during retrograde ureteroscopic lithotripsy. For instance, during 
percutaneous nephrolithotomy, stone measurement could aid the surgeon in determining whether to proceed with ultrasonic $v s$ laser lithotripsy and decrease the incidence of retained fragments and prolonged procedures, which are common publicly reported complications after this procedure. ${ }^{16}$ This technology may also have applications to upper tract malignancy (e.g., determination of urothelial tumor size and area of tumor involvement) and stricture disease (e.g., measurement of ureteral caliber before and after endoscopic treatment). There may also be relevance to gastrointestinal endoscopy, where recent studies have described ruler-like adjuncts that could be installed onto an endoscope,${ }^{17}$ unlike this study in which measurements were entirely digital. Alongside technical studies to ensure accurate measurement of nonstone targets, these examples highlight the broad applicability of real-time measurement technologies in endoscopic procedures.

\section{Conclusions}

Integrating real-time stone measurement during live ureteroscopy is safe and feasible. Digital measurement has a small impact on the time required for stone manipulation. Although during live surgery several measurements may be required, the additional time is modest relative to total operating time. These findings support prospective clinical trials of digital stone measurement to enhance intraoperative decision-making during ureteroscopy.

\section{Author Disclosure Statement}

K.K., T.A., R.E.N.B., S.L., J.S.W., D.P., and D.S. declare that they have no competing financial interests. B.R.M. is a consultant for Boston Scientific Corporation, but this research was not related.

\section{Funding Information}

No funding was received for this article.

\section{References}

1. De Coninck V, Keller EX, Somani B, et al. Complications of ureteroscopy: A complete overview. World J Urol 2019 [Epub ahead of print]; DOI: 10.1007/s00345-019-03012-1.

2. Cornu JN, Herrmann T, Traxer O, Matlaga B. Prevention and management following complications from endourology procedures. Eur Urol Focus 2016;2:49-59.

3. Patel N, Chew B, Knudsen B, Lipkin M, Wenzler D, Sur RL. Accuracy of endoscopic intraoperative assessment of urologic stone size. J Endourol 2014;28:582-586.

4. Joice GA, Lim S, Ludwig WW, Gorin MA, Stoianovici D, Matlaga BR. MP32-21 clinical utility of endoscopic stone measurement. J Endourol 2018;32:A329-A330.

5. Ludwig WW, Lim S, Stoianovici D, Matlaga BR. Endoscopic stone measurement during ureteroscopy. J Endourol 2018;32:34-39.

6. Ward JB, Feinstein L, Pierce C, et al. Pediatric urinary stone disease in the United States: The Urologic Diseases in America Project. Urology 2019;129:180-187.
7. Geraghty RM, Jones P, Somani BK. Worldwide trends of urinary stone disease treatment over the last two decades: A systematic review. J Endourol 2017;31:547-556.

8. Assimos D, Krambeck A, Miller NL, et al. Surgical management of stones: American Urological Association/Endourological Society Guideline, Part I. J Urol 2016;196: 1153-1160.

9. Assimos D, Krambeck A, Miller NL, et al. Surgical management of stones: American Urological Association/ Endourological Society Guideline, Part II. J Urol 2016;196: 1161-1169.

10. Khaleel SS, Borofsky MS. Innovations in disposable technologies for stone management. Urol Clin North Am 2019; 46:175-184.

11. Cordes J, Teske L, Nguyen F, Pinkowski W, Sievert KD, Vonthein R. A comparison between an in vitro ureteroscopic stone size estimation and the stone size measurement with the help of a scale on stone baskets. World J Urol 2016;34:1303-1309.

12. Cordes J, Nguyen F, Pinkowski W, Merseburger AS, Ozimek T. A new automatically fixating stone basket (2.5F) prototype with a Nitinol spring for accurate ureteroscopic stone size measurement. Adv Ther 2018;35: 1420-1425.

13. Ansari MS, Goel A, Karan SC, Aron M. Holmium: YAG laser rescue for a stuck stone basket. Int Urol Nephrol 2002; 34:463-464.

14. Tracy CR, Ghareeb GM, Paul CJ, Brooks NA. Increasing the size of ureteral access sheath during retrograde intrarenal surgery improves surgical efficiency without increasing complications. World J Urol 2018;36:971-978.

15. Chawla SN, Chang MF, Chang A, Lenoir J, Bagley DH. Effectiveness of high-frequency holmium:YAG laser stone fragmentation: the "popcorn effect". J Endourol 2008;22: 645-650.

16. Patel NH, Schulman AA, Bloom JB, et al. Device-related adverse events during percutaneous nephrolithotomy: Review of the manufacturer and user facility device experience database. J Endourol 2017;31:1007-1011.

17. Kume K, Watanabe T, Yoshikawa I, Harada M. Endoscopic measurement of polyp size using a novel calibrated hood. Gastroenterol Res Pract 2014;2014:714294.

Address correspondence to: Kevin Koo, MD, MPH, MPhil Department of Urology Johns Hopkins University School of Medicine 600 North Wolfe Street Baltimore, MD 21287 USA

E-mail:kkoo@jhmi.edu

$$
\begin{gathered}
\text { Abbreviations Used } \\
\mathrm{SD}=\text { standard deviation } \\
\mathrm{UPJ}=\text { ureteropelvic junction }
\end{gathered}
$$

